\title{
Rituximab: a novel treatment for refractory Riedel's thyroiditis
}

Leanne Hunt, Barney Harrison, Matthew Bull, Tim Stephenson and Amit Allahabadia

Sheffield Teaching Hospitals, Sheffield, UK
Correspondence

should be addressed

to L Hunt

Email

leanne.hunt@sheffield.ac.uk

\section{Summary}

This case report reviews the rare condition of Riedel's thyroiditis via a patient case. The report highlights the difficulties that one may encounter when managing such a case in regards to patient symptoms, side effects of medications and the relapsing nature of the condition. The case report also highlights novel treatment in the treatment of Riedel's thyroiditis, rituximab, how this works and the resolution of symptoms that we have achieved with our patient on this treatment.

\section{Learning points:}

- Riedel's thyroiditis is characterised by chronic inflammation, which causes dense fibrosis in the thyroid gland.

- Riedel's thyroiditis can present with neck pain, dysphagia and dyspnoea with a firm, non-tender mass found on examination.

- Riedel's thyroiditis is part of the IgG4-related systemic disorders.

- Rituximab is a monoclonal antibody that works against the protein CD20.

\section{Background}

This case is important as it highlights the difficulties in managing complex Riedel's thyroiditis patients. The case also highlights the novel use of rituximab in the treatment of a patient with complex refractory Riedel's thyroiditis. As this is a rare condition, this case will provide information to readers on the success we have had with this treatment and shed light on the mechanism by which rituximab works.

\section{Case presentation}

\section{Background}

Riedel's thyroiditis (RT) is a rare condition characterised by a chronic inflammatory process in which one or both thyroid lobes are replaced by dense fibrosis that may extend beyond the thyroid capsule and involve adjacent neck structures. These features differentiate the disease from other disorders such as Hashimoto's thyroiditis.
A case series of 57000 thyroidectomies performed between 1920 and 1984 at the Mayo clinic revealed an incidence of Riedel's of $1 / 1540$ on post-operative histology (1). Peak onset occurs between the ages of 30 and 50 years, and it is four times more common in women $(2,3)$.

Typical presentation is with anterior neck pain, dysphagia, dyspnoea and a feeling of suffocation (particularly on lying down) due to oesophageal or tracheal compression $(1,4)$. Examination findings are typically a firm, non-tender thyroid mass that commonly extends to the surrounding tissues. Recurrent laryngeal nerve and cervical sympathetic trunk involvement and superior vena cava obstruction can occur $(5,6)$. Fibrous mediastinitis and occlusive vasculitis have also been reported (7).

The majority of patients with RT are euthyroid. Thyroid dysfunction when present usually manifests as hypothyroidism often associated with elevated antibody 
titres against thyroglobulin (TG-Ab) and thyroperoxidase (TPO-Ab) (6). Thyroid dysfunction also occurs due to extensive replacement of the thyroid by non-functioning fibrous tissue. The Cleveland clinic review of 185 patients found that $64 \%$ of patients were euthyroid, $32 \%$ hypothyroid and $4 \%$ hyperthyroid (8).

The course of the disease is variable, from self-limiting to more aggressive forms in which one or more treatments are required for suppression of the inflammatory process and its effects on structures in the neck, mediastinum and beyond. We report a patient with a protracted course of RT over a number of years requiring multiple interventions and the novel use of the biological agent, rituximab for its control.

\section{Case}

In June 2007, a 45-year-old female presented with thyroid enlargement. Fine-needle aspirate biopsy (FNAB) revealed a lymphoid aspirate. Blood tests revealed marked elevation of thyroid peroxidase antibodies $(1850 \mathrm{U} / \mathrm{mL}$ (0-50)), slight elevation of thyrotrophin-stimulating hormone (TSH) of 7.14 U/L (0.35-5.5) but normal serumfree thyroxine (fT4) concentration of $12.05 \mathrm{pmol} / \mathrm{L}$ (9-24). A diagnosis of Hashimoto's thyroiditis was made.

In December 2007, rapid growth and swelling of her neck mass occurred with associated compressive symptoms. Concerns were raised about the possibility of Hashimoto's thyroiditis-associated lymphoma, and an open thyroid biopsy was undertaken. Histology revealed an abundance of lymphoid and eosinophil cells, bands of birefringent, sclerotic collagen with crushed cellular infiltrate insinuating between the collagen bands. The case was discussed at the thyroid multi-disciplinary team (MDT) meeting and RT diagnosed.

The neck swelling and compressive symptoms initially responded well to treatment with high-dose dexamethasone $10 \mathrm{mg}$ and $20 \mathrm{mg}$ tamoxifen once-daily (OD), but these medications were poorly tolerated due to nausea and fluid retention and subsequently stopped. The patient was referred in March 2008 to the endocrine surgery team in a tertiary referral centre. Clinical features included dysphagia, recent-onset hoarseness and a moderate-sized, hard swelling in the centre of the neck with retrosternal extension. CT scan (Fig. 1) revealed mild compression of the cervical trachea due to thyroid enlargement. Video laryngoscopy revealed partial weakness of the right vocal cord. The patient was hypothyroid (TSH 7.9 IU/L (0.27-4.2 IU/L), fT4 $11.3 \mathrm{pmol} / \mathrm{L} \quad(12-22 \mathrm{pmol} / \mathrm{L}))$ and commenced on $75 \mu \mathrm{g}$ of levothyroxine.

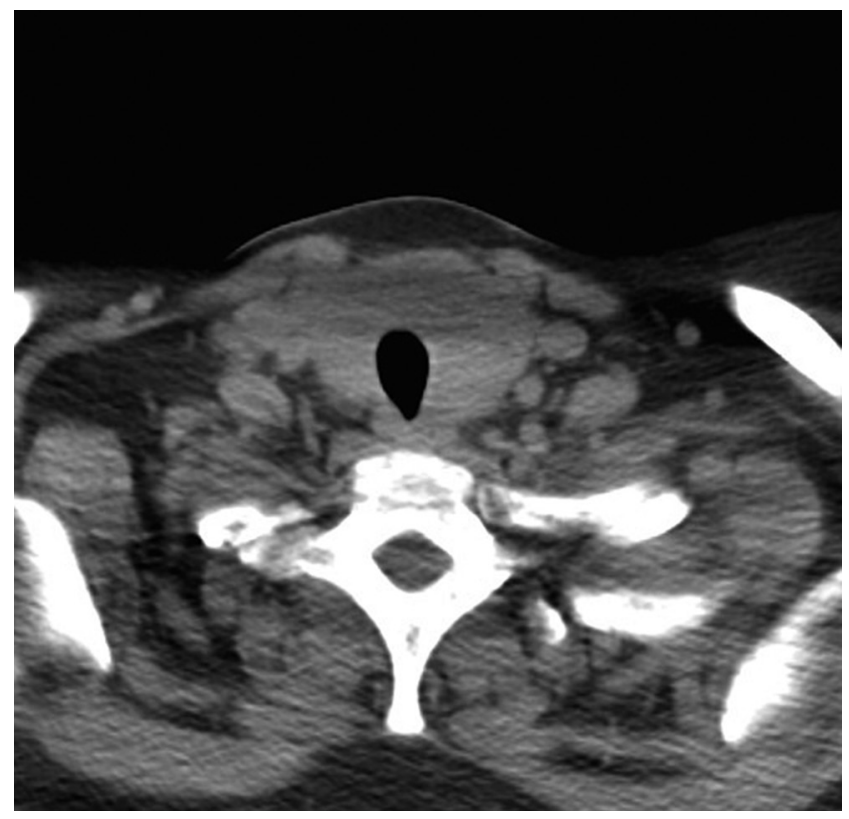

Figure 1

CT neck. Both lobes of the thyroid are enlarged, slightly more marked on the left, causing mild tracheal narrowing with a reduction in the transverse diameter of the trachea to $13 \mathrm{~mm}$.

In June 2008, the patient reported that the goitre had increased in size and had become painful. Clinical examination confirmed a tender, hard neck mass. Blood tests revealed an elevated TSH of $8.44 \mathrm{U} / \mathrm{L}$ so the levothyroxine dose was increased to $100 \mu \mathrm{g}$ OD. MRI (Fig. 2) confirmed that the thyroid mass had increased in size with further compromise of the tracheal lumen. Tamoxifen therapy (20 mg OD) was reinstated.

In October 2008, due to worsening neck pain and swelling, repeat MRI was performed. Further progression of the mass on serial cross-sectional imaging was noticed despite the current medical therapy. A subtotal thyroidectomy was performed. Surgery was difficult due to the presence of dense fibrosis making identification of the thyroid gland difficult. Histology from the surgery confirmed features of RT.

Postoperatively, the patient was started on a reducing regimen of dexamethasone, initially $2 \mathrm{mg}$ per day. There was an excellent therapeutic response and as her hypothalamic-pituitary-adrenal (HPA) axis remained intact, dexamethasone and tamoxifen therapy were withdrawn.

In June 2009, symptoms of neck pain, dysphagia and shortness of breath recurred, so tamoxifen $20 \mathrm{mg}$ OD was reinstated. A repeat MRI scan confirmed enlargement of the residual thyroid mass from $4 \times 3.3 \mathrm{~cm}$ to $6 \times 4 \mathrm{~cm}$ (Fig. 3). 


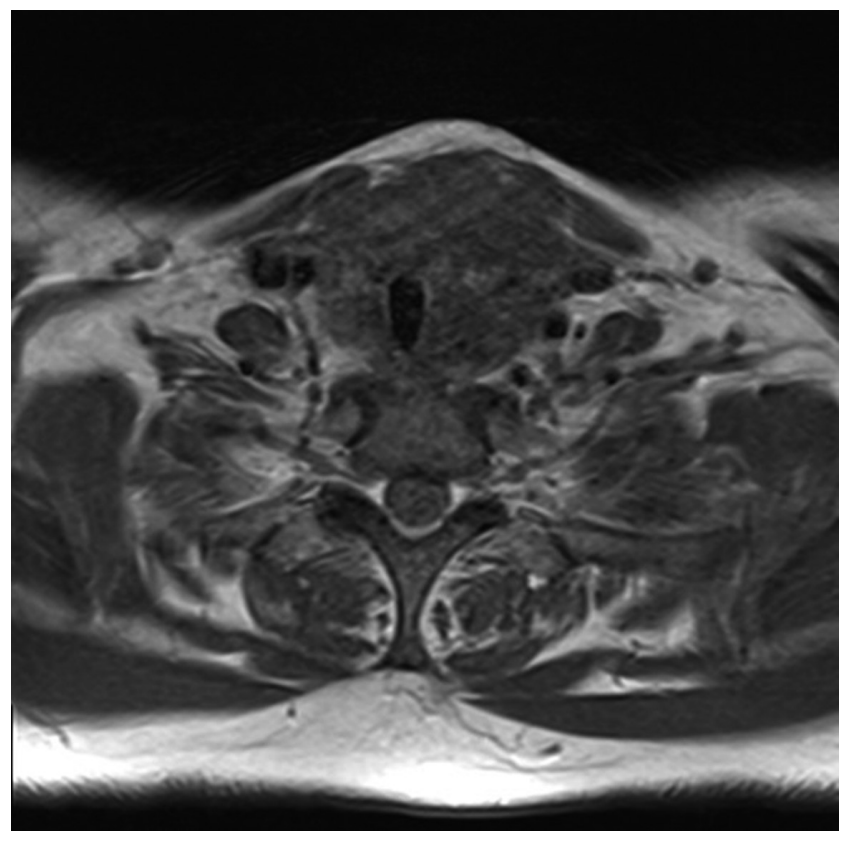

Figure 2

T1-weighted MR with contrast. Isointense thyroid gland with reduced enhancement following contrast administration consistent with Riedel's thyroiditis. The degree of tracheal compression has increased with a minimum mediolateral diameter of $11 \mathrm{~mm}$.

Treatment options were discussed with the patient including observation alone, low-dose dexamethasone with tamoxifen or raloxifene or repeat surgery.

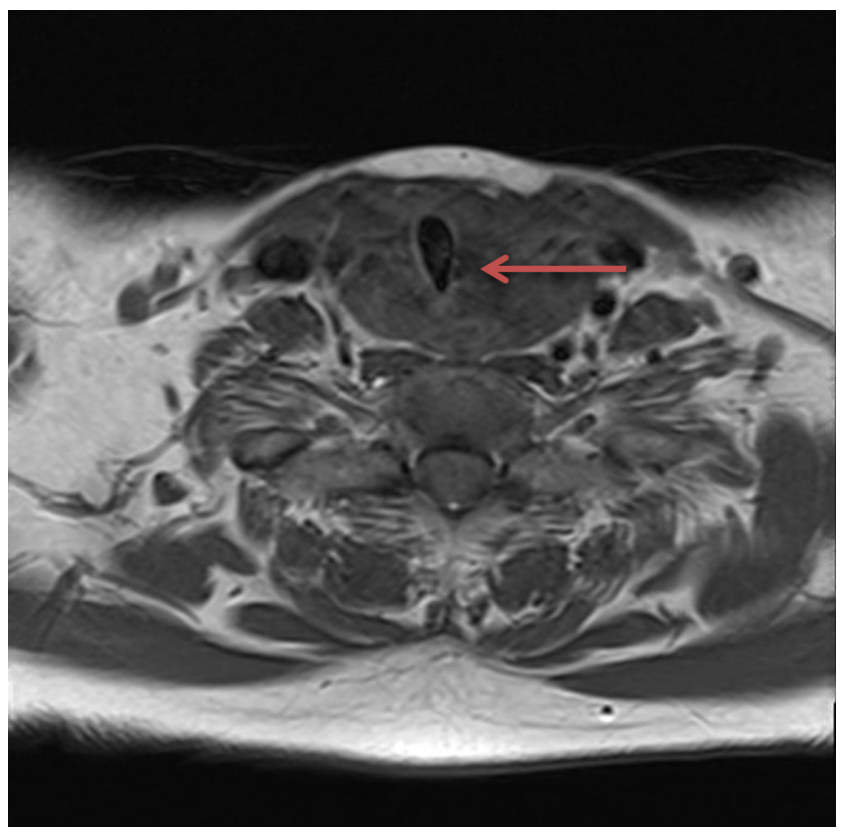

Figure 3

T1-weighted MR with contrast. T1-weighted image showing enlargement of the homogenous thyroid mass to $6 \times 4 \mathrm{~cm}$, associated with tracheal narrowing at the sternal notch (arrow).
Dexamethasone and raloxifene therapy was selected, the latter chosen because of the side effects experienced with tamoxifen. Unfortunately, she developed nausea with raloxifene and in view of the previously documented efficacy, tamoxifen was restarted.

Between 2010 and 2012, symptoms remained stable with no significant change in size of the residual thyroid mass on imaging.

In April 2013, the patient reported an increase in neck pain and enlargement of the residual thyroid mass that was confirmed on clinical examination. Raloxifene was recommenced as it was thought that tamoxifen had lost its efficacy. Over the course of the year, dexamethasone (between 2 and $10 \mathrm{mg}$ per day) was titrated according to her symptoms. The patient developed a Cushingoid appearance but described improvement of her compressive symptoms. In late 2013, she noticed further thyroid enlargement and neck tightness requiring $10 \mathrm{mg}$ of dexamethasone for symptom control.

In January 2014, whilst on dexamethasone therapy (9 mg OD), the patient underwent repeat MRI, which confirmed further thyroid enlargement.

As RT is known to be part of the syndrome, IgG4related systemic disease (IgG4-RSD), the patient was given intravenous (IV) rituximab therapy. Prior to treatment, her serum IgG4 level was measured at $4.43 \mathrm{~g} / \mathrm{L}$ (6.0-16.0). She received monthly infusions of $1 \mathrm{~g}$ IV rituximab with $100 \mathrm{mg}$ IV methylprednisolone between May and

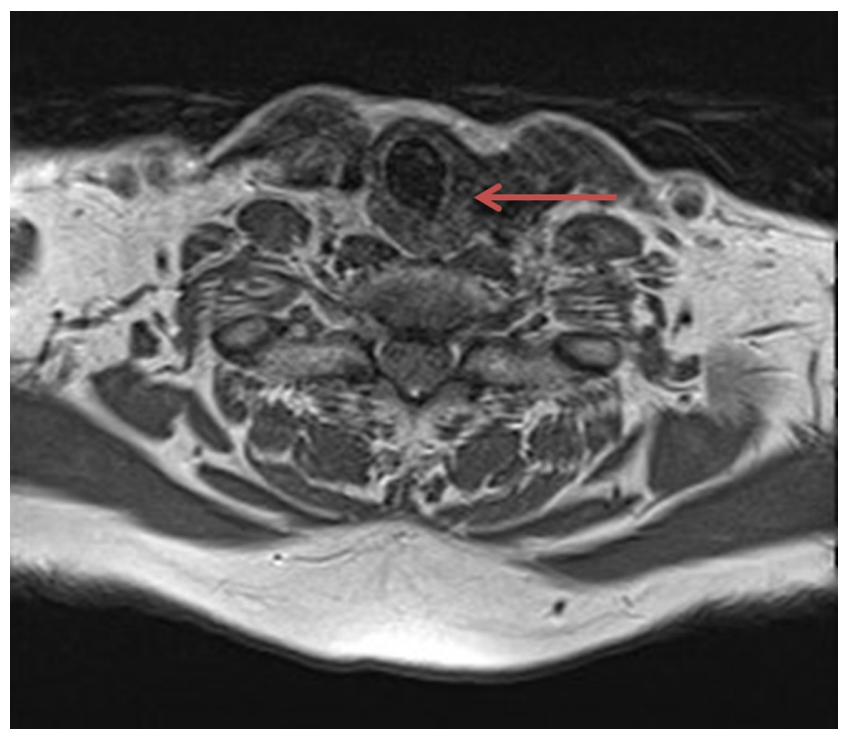

Figure 4

T1-weighted MR with contrast. The amount of disease encasing the left carotid sheath has decreased from $21 \mathrm{~mm}$ thickness to $15 \mathrm{~mm}$ thickness with no significant tracheal compression. 
July 2014. She remained on raloxifene and dexamethasone therapy was stopped.

Following rituximab infusions, the symptoms of neck pain, shortness of breath and dysphagia have completely resolved and remained quiescent at 30 months post treatment. Clinical and radiological examination confirms reduction in the size of the residual thyroid tissue. MRI shows that the mass encasing the left carotid sheath has decreased from $21 \mathrm{~mm}$ to $15 \mathrm{~mm}$ thickness (Fig. 4). The patient remains on raloxifene $60 \mathrm{mg}$ twice daily and levothyroxine $150 \mu \mathrm{g} / \mathrm{day}$.

\section{Investigation}

The patient had multiple investigations during the course of her treatment. She had multiple thyroid function tests and various radiological investigations. Initial investigations once referred to Sheffield teaching hospitals included:

(1) March 2008: CT scan (Fig. 1) revealed mild compression of the cervical trachea due to thyroid enlargement. Video laryngoscopy revealed partial weakness of the right vocal cord. The patient was hypothyroid (TSH: 7.9 IU/L (0.27-4.2 IU/L), fT4: $11.3 \mathrm{pmol} / \mathrm{L}(12-22 \mathrm{pmol} / \mathrm{L}))$ and commenced on $75 \mu \mathrm{g}$ of levothyroxine.

(2) June 2008: Blood tests revealed an elevated TSH of $8.44 \mathrm{U} / \mathrm{L}$ so the levothyroxine dose was increased to $100 \mu \mathrm{g}$ OD. MRI (Fig. 2) confirmed that the thyroid mass had increased in size with further compromise of the tracheal lumen. Tamoxifen therapy (20 mg OD) was reinstated.

(3) October 2008: Repeat MRI was performed. Further progression of the mass on serial cross-sectional imaging was noticed despite the current medical therapy. A subtotal thyroidectomy was performed.

(4) June 2009: Repeat MRI scan confirmed enlargement of the residual thyroid mass from $4 \times 3.3 \mathrm{~cm}$ to $6 \times 4 \mathrm{~cm}$ (Fig. 3).

(5) Between 2010 and 2012: Remain clinically stable with no changes on MR imaging.

(6) January 2014: Repeat MRI showed further thyroid enlargement - rituximab started.

(7) July 2014: MRI shows that the mass encasing the left carotid sheath has decreased from $21 \mathrm{~mm}$ to $15 \mathrm{~mm}$ thickness (Fig. 4). Unfortunately, as her clinical picture has remained stable and improved with the rituximab therapy, we do not have any further imaging.

\section{Treatment}

During the course of her treatment for RT, the patient had multiple treatments including steroid therapy, raloxifene and tamoxifen therapy. Unfortunately, her RT was refractory to these treatments, and she suffered side effects. The decision to use rituximab was due to the refractory nature of her condition and continued thyroid enlargement despite treatment. Dosages of medications used:

- Tamoxifen: $20 \mathrm{mg}$ OD.

- Levothyroxine: Up to $75 \mu \mathrm{g}$ OD.

- Dexamethasone: Up to $9 \mathrm{mg}$ OD.

- Raloxifene: $60 \mathrm{mg}$ OD.

- Rituximab: $1 \mathrm{~g}$ IV once monthly for three months with $100 \mathrm{mg}$ methylprednisolone cover.

\section{Outcome and follow-up}

The initial referral to the endocrinology team at Sheffield Teaching Hospitals was June 2008. She was seen at various points over the following years, usually on a 3-6 monthly basis. This has now extended since completion of rituximab therapy in 2014.

\section{Discussion}

Although RT is generally a slowly progressive, self-limiting condition, there can be significant morbidity and mortality associated with the condition (7). The morbidity that can occur from local compressive symptoms is significant. Evidence has suggested that one-third of patients with RT will develop fibrosing disorders in other organs over a tenyear period (9), so with this in mind patients do undergo treatment for RT.

Core or incision biopsy is most likely required to confirm the diagnosis. Imaging of the thyroid, neck and mediastinal structures will reveal the extent of the disease. ${ }^{18} \mathrm{~F}$ positron emission tomography (FDG-PET) scans confirm the active inflammatory process (10).

Hypothyroidism or hypoparathyroidism are managed with levothyroxine, calcium and vitamin D replacement. Surgery is often difficult due to the fibrotic obliteration of resectional planes with increased risk of injury to adjacent structures and reports of recurrence post surgery (11). Even with limited surgical intervention, treated by expert surgical teams, seven of 18 (39\%) patients had complications (permanent vocal cord paralysis, hypoparathyroidism) (12). Surgery is therefore only 
indicated for significant tracheal and oesophageal compression and should be limited to obtaining symptomatic relief rather than complete disease clearance.

Medical treatment of RT includes glucocorticoids, tamoxifen and raloxifene. Due to the rarity of the condition, these interventions have not been fully validated in controlled trials.

Glucocorticoids are of benefit in early disease, particularly in patients with active inflammation (13). They are generally regarded as first line therapy and there have been several reports showing reduction in the size and the mass and improvement in symptoms. Initial prednisolone doses of up to $100 \mathrm{mg}$ OD have been reported but the maintenance dose is usually between 15 and $60 \mathrm{mg}$ (4). Dysphonia and recurrent laryngeal nerve involvement may resolve with glucocorticoid therapy (14). However the response rate is variable. Active smokers with more aggressive fibrotic progression seem to require a longer duration of treatment with repeated courses of glucocorticoid therapy (13) and may relapse after withdrawal of glucocorticoid therapy. Failure of glucocorticoid therapy determines the need for other treatments to supress disease activity.

Tamoxifen, a selective oestrogen receptor modulator (SERM), can be used as either a monotherapy (in patients refractory to glucocorticoids) or as adjunctive therapy to allow a reduced dose of glucocorticoids. It decreases the expression of TGF- $\beta$ resulting in inhibition of fibroblast proliferation and collagen production (15). There is evidence of reduction in the size of fibrosis and reduction in symptoms in some but not all cases of RT with tamoxifen (16). Doses of $10-30 \mathrm{mg}$ twice daily have been used (4). Side effects are common with tamoxifen treatment, and these include hot flushes, menstrual irregularity and an increased endometrial carcinoma risk. Our patient did develop side effects but was able to manage these in the long term.

Unfortunately, despite remaining stable for over two years, symptoms in our patient returned and she reported distressing side effects. Treatment was changed to an alternative SERM, raloxifene, unlike tamoxifen, raloxifene is not associated with a risk of endometrial carcinoma risk, but there are no available studies to demonstrate its effect on pathological fibrosis.

For patients with symptomatic RT refractory to glucocorticoid and SERM therapy, there are few alternative treatments. Mycophenalate mofetil has been used in conjunction with high-dose prednisolone $(100 \mathrm{mg} /$ day). Levy and coworkers reported significant reduction in the size of a thyroid mass in a refractory case (16). Mycophenalate has potentially serious side effects, it is immunosuppressive and pancytopenia, gastrointestinal bleeding, renal failure and lymphoproliferative disorders are described with its use.

RT is part of the spectrum of IgG4-related systemic disorders (IgG4-RSD) of unknown aetiology that share specific clinical, serological and histopathological features (17) including lymphoplasmacytic infiltrate, fibrosis, eosinophilic and obliterative infiltrate (18).

In IgG4-RSD glucocorticoid therapy is effective particularly if extensive fibrosis has not been encountered. Mycophenalate and azathioprine have also been used.

Rituximab is a monoclonal antibody that works against the protein $\mathrm{CD} 20$ primarily found on the surface of B cell lymphocytes. Rituximab destroys B cells and is therefore used to treat conditions that are characterised by excessive numbers of B cells such as severe Grave's opthalmopathy (19) and relapsed Grave's disease (20). More recently, it has been used in patients with known IgG4-RSD resistant to glucocorticoid therapy (21). A raised serum IgG4 level is normally found with IgG4RSD; in contrast, the serum IgG4 level in our patient was $4.43 \mathrm{~g} / \mathrm{L}$ (6.0-16.0). Levels within the normal range are described in other IgG4-RSD such as immunoglobulin G4-associated cholangitis (22).

The response to rituximab in our patient is similar to the effect seen in a case report by Shui-Boon and coworkers (7), the only reported use of rituximab in refractory RT. Their patient had prompt and robust symptom relief with rituximab, a normal serum level of IgG4 at the end of treatment. Given the dramatic improvement in both these patients' symptoms and the disease regression seen on cross-sectional imaging, rituximab should be considered as a therapeutic option in patients in whom conventional treatments for RT are poorly tolerated or ineffective.

Declaration of interest

The authors declare that there is no conflict of interest that could be perceived as prejudicing the impartiality of the research reported.

\section{Funding}

This research did not receive any specific grant from any funding agency in the public, commercial or not-for-profit sector.

Patient consent

Patient consent has been obtained. 


\section{Author contribution statement}

Leanne Hunt: primary author; Barney Harrison: review and edit of report, consultant surgeon responsible for patient; Matthew Bull: provision and review of radiological images; Tim Stephenson: review of patients histological findings; Amit Allahabadia: review and edit of report, consultant endocrinologist responsible for patient.

\section{References}

1 Hay ID. Thyroiditis: a clinical update. Mayo Clinic Proceedings 1985 60 836-843.

2 Woolner LB, McConahey WM \& Beahrs OH. Invasive fibrous thyroiditis (Riedel's struma). Journal of Clinical Endocrinology and Metabolism 195717 201-220.

3 Fatourechi MM, Hay ID, McIver B, Sebo TJ \& Fatourechi V. Invasive fibrous thyroiditis (Riedel's thyroiditis): the Mayo Clinic Experience 1976-2008. Thyroid 201121 765-772. (https://doi.org/10.1089/ thy.2010.0453)

4 Hennessey JV. Riedel's thyroiditis: a clinical review. Journal of Clinical Endocrinology and Metabolism 201196 3031-3041. (https://doi. org/10.1210/jc.2011-0617)

5 Yasmeen T, Khan S, Patel SG, Reeves WA, Gonsch FA, de Bustros A \& Kaplan EL. Riedel's thyroiditis: a report of a case complicated by spontaneous hypoparathyroidism, recurrent laryngeal nerve, and Horner's syndrome. Journal of Clinical Endocrinology and Metabolism 200287 3543-3547.

6 Papi G \& LiVolsi VA. Current concepts on Riedel's thyroiditis. American Journal of Clinical Pathology 200421 S50-S63.

7 Soh SB, Pham A, O'Hehir RE, Cherk M \& Topliss DJ. Novel use of Rituximab in a case of Riedel's thyroiditis refractory to glucocorticoids and tamoxifen. Journal of Clinical Endocrinology and Metabolism 201398 3543-3549. (https://doi.org/10.1210/jc.20124050)

8 Schwaegerle SM, Bauer TW \& Esselstyn CB Jr. Riedel's thyroiditis. American Journal of Clinical Pathology 198890 715-722. (https://doi. org/10.1093/ajcp/90.6.715)

9 Oguz KK, Kiratli H, Oguz O, Cila A, Oto A \& Gokoz A. Multifocal fibrosclerosis: a new case report and review of the literature. European Radiology 200212 1134-1138. (https://doi.org/10.1007/s00330-0011158-4)

10 Kotilainen P, Airas L, Kojo T, Kurki T, Kataja K, Minn H \& Nuutila P. Positron emission tomographyas an aid in the diagnoisis and follow-up of Riedels thyroiditis. European Journal of Internal Medicine 200415 186-189. (https://doi.org/10.1016/j.ejim.2004.03.002)
11 Zimmermann-Belsing T \& Feldt-Rasmussen U. Riedel's thyroiditis: an autoimmune or primary fibrotic disease? Journal of Internal Medicine 1994235 271-440. (https://doi.org/10.1111/j.1365-2796.1994. tb01071.x)

12 Fatourechi MM, Hay ID, McIver B, Sebo TJ \& Fatourechi V. Invasive fibrous thyroiditis (Riedel's thyroiditis): the Mayo Clinic Experience 1976-2008. Thyroid 201121 765-772. (https://doi.org/10.1089/ thy.2010.0453)

13 Vaidya B, Harris PE, Barrett P \& Kendall-Taylor P. Corticosteroid therapy in Riedel's thyroiditis. Postgraduate Medical Journal 199773 817-819. (https://doi.org/10.1136/pgmj.73.866.817)

14 Bagnasco M, Passalacqua G, Pronzato C, Albano M, Torre G \& Scordamaglia A. Fibrous invasive (Riedel's) thyroiditis with critical response to steroid treatment. Journal of Endocrinological Investigation 199518 305-307.

15 Gragnani A, Warde M, Furtado F \& Ferreira LM. Topical Tamoxifen therapy in hypertrophic scars or keloids in burns. Archives of Dermatological Research 2010302 1-4. (https://doi.org/10.1007/ s00403-009-0983-1)

16 Levy JM, Hasney CP, Friedlander PL, Kandil E, Occhipinti EA \& Kahn MJ. Combined mycophenolate mofetil and prednisolone therapy in tamoxifen- and prednisolone-resistant Riedel's thyroiditis. Thyroid 201020 105-107. (https://doi.org/10.1089/thy.2009.0324)

17 Stone JH, Zen Y \& Deshpande V. IgG4-related disease. New England Journal of Medicine 2012366 539-551. (https://doi.org/10.1056/ NEJMra1104650)

18 Cheuk W \& Chan JK. IgG4-related sclerosing disease: a critical appraisal of an evolving clinicopathologic entity. Advances in Anatomic Pathology 201017 303-332. (https://doi.org/10.1097/ PAP.0b013e3181ee63ce)

19 El Fassi D, Nielsen CH, Hasselbalch HC \& Hegedus L. Treatment resistant severe, active Graves' ophthalmology successfully treated with B lymphocyte depletion. Thyroid 200616 709-710. (https://doi. org/10.1089/thy.2006.16.709)

20 Heemstra KA, Toes RE, Sepers J, Pereira AM, Corssmit EP, Huizinga TW, Romijn JA \& Smit JW. Rituximab in relapsing Graves' disease: a phase II study. European Journal of Endocrinology 2008159 609-615. (https://doi.org/10.1530/EJE-08-0084)

21 Khosroshahi A \& Stone JH. Treatment approaches to IgG4-related systemic disease. Current Opinion in Rheumatology 201123 67-71. (https://doi.org/10.1097/BOR.0b013e328341a240)

22 Ghazale A, Chari ST, Zhang L, Smyrk TC, Takahashi N, Levy MJ, Topazian MD, Clain JE, Pearson RK, Petersen BT, et al. Immunoglobulin G4-associated cholangitis: clinical profile and response to therapy. Gastroenterology 2008134 706-715. (https://doi. org/10.1053/j.gastro.2007.12.009)

Received in final form 24 December 2017

Accepted 12 January 2018 